\title{
Concept of the Social Design in Public Spaces Practice - cases of Berlin and Taipei
}

\author{
Shiau-Ting Wang $a,{ }^{*}$ and Sheng-Ming Wang $b$ \\ a,bInteraction Design Department, National Taipei University of Technology \\ Taipei, Taiwan \\ E-mail: christinewang1990@gmail.com, ryan5885@mail.ntut.edu.tw
}

The study aims to compare two social design concepts that take place in Taipei and Berlin by the case study. The first case focus on the technology applied in the solution, the historical area located in Taipei, it used AR, chatbot, and web VR to let the users in-depth experience of the background story. On the other hand, Berlin's case was done under design oriented, it follows user-center design to define the problem and focus on the user experience, finally comes up with a conceptual App, these cases we chose are both based on the concept of increasing the connectivity between people and regions. This study follows the process of action research, and the innovation model proposed by IDEO was used to analysis these two cases, it has three essential perspectives, "Feasibility", "Desirability" and "Viability". This research is currently in the process, but we expect the result could improve the practice of social design in public spaces, as the conclusion, we found out that it needs not only balance integration of technology and design content but also include a business field to make the service system more complete.

Keywords: Social Design, Action Research, Innovation Model, Interdisciplinary 


\section{Introduction}

Social design can be done in many different ways, the scope is very broad, and each field tries to think and solve problems with its own familiar point of view, so we think about that is it easy to cause defects in the design practice due to a single discipline? Maybe to implementing interdisciplinary integration for social design in public space could make more contribution. The cases in this study were the course that author taken and collected it as the data then.

\section{Literature Review}

\subsection{Action research}

The action research was proposed in 1944 by Kurt Lewin, it's a kind of qualitative research, they're three main primary data collection ways, "interview", "observations", and "document analysis" [5], such approaches include grounded theory, ethnography, and case study, the efforts all yield observable effects on practice [2]. As you can see Figure.1, this method starts from identify the problem, develop a plan of the action, collect the data and analyze them, it's a circular and combines theory and practice [1]. The primary reason for engaging in action research is to assist the "actor" in improving and refining his or her actions [5], the actor of this study is the concept of social design in public spaces practice, which take place in Taipei and Berlin.

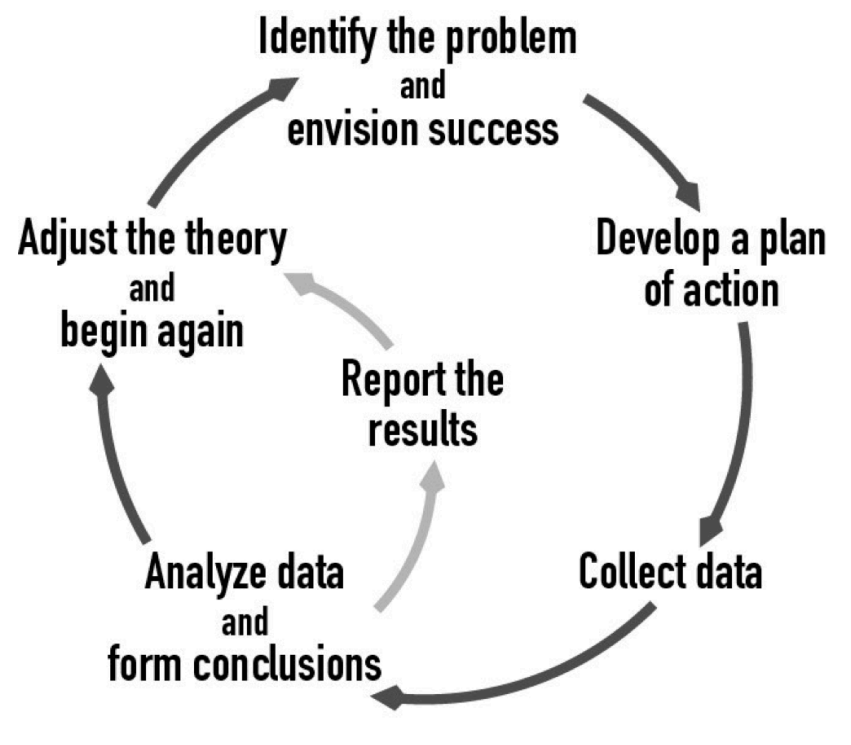

Figure. 1: Process of action research. (source from thecreativeeducation.com)

\subsection{Social Design Case Study - City Yeast}

City yeast is a social design project that initiated in Taipei, the start of City yeast, "plan global", is proposed by AUGA Design (the name of company) in 2003; the idea is not aimed to start with business, but to return to the value of design, to think about human needs and social problems, and to develop not only products, but also concepts, systems or behavior patterns. The idea is to broaden the design perspective. The project call "City yeast", through realizing the relationship and possibilities between ourselves and our living environment, they decided to 
create a lot of creative and interesting yeast in the city, and like yeast revitalization, to build a non-globalized local character, so that people will have a deeper link with the city and fall in love with the place they live [7]. The concept of City yeast is very similar to the cases that we collected, people start to think about the relationship between the region and human, want to make the stronger connectivity. This case was a ten-year project, so it includes many levels of design, and also involves people from various fields, including domestic and foreign graphic, spatial and industrial designers, directors, poets, and academic researchers. It was presented through many types of way but mostly traditional way, like physical interactive exhibition, internet, and book publication.

\subsection{Innovation Model}

Innovation model was proposed by IDEO, they talked about who design support innovation, there are three essential perspectives, and the yellow part is the innovation, the sweet spot is the convergence of what is desirable from a human point of view with what is technologically feasible and economically viable. (as Figure.2), (1) "Feasibility" - is an engineering-focused discussion around platforms, architecture, processes, skills, and tools, (2) "Desirability" - is a design-focused discussion around customers, aesthetics, function, and form. For design-focused products, the focus might be on context, culture, customer goals, product experience, design aesthetics and interactions, and (3) "Viability" - is a business-focused discussion around marketing and finance, an analytical debate around the business, rather than the core user experience. Financial metrics and market sizes become the dominating point of discussion.

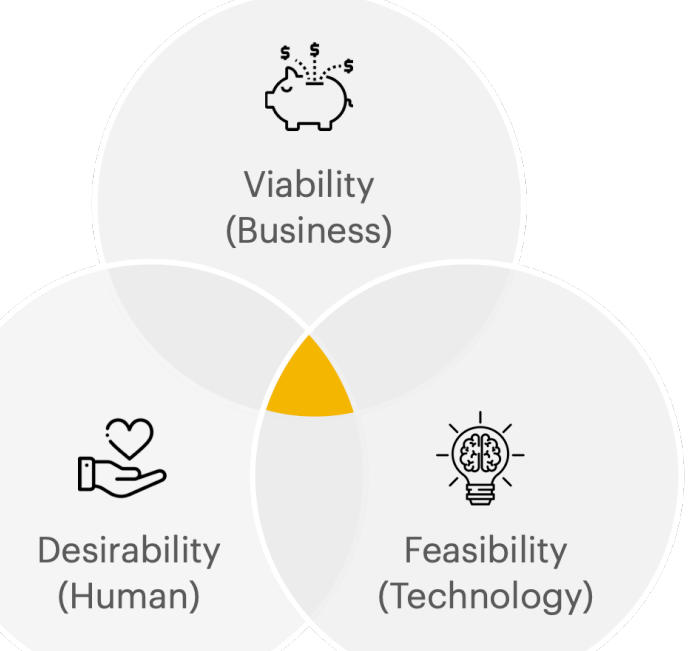

Figure. 2: Three essential perspectives of innovation provide by IDEO. (drawn by this research)

\section{Research Method \& Process}

This study follow the process of action research, and the cases we collected were both done in school curriculum, so to distinguish the potential purposes of action research is really important [3]. However, the two courses lead to different directions, the one in Taipei is more technology oriented, Berlin side is more focus on the design oriented and creativity, therefore, we provide the assumption which will talk about it later. 


\subsection{Identify the problem}

We hope to find out through this method whether the social design in public space is easy to cause defects in the design practice due to a single discipline? And also want to know if the integration of multiple disciplines contributes to the social design of public spaces.

\subsection{Plan of Action}

From a social design point of view, we will collect tech oriented and design oriented cases, make the comparison and analysis them based on the exploration of how to increase the connectivity between people and regions. Technology oriented was done in Taipei by the student from interaction design department, and the design oriented project was done in Berlin by the student from interface design department.

\subsection{Data Collection}

The reason why we choose this two cases is because of the results of social design in public spaces from different cultural backgrounds and regions. Details of two concept will be explained in the following sections.

\subsubsection{Bridging Conversations with the Past \& Present (Taipei)}

This concept called "Bridging Conversations with the Past \& Present", the region they choose is a historical area named Dadaocheng, it was prosperous in the 1920s but is now in decline. The current way of exploring is passively look up information before you go there, and it is impossible to understand the historical and cultural background through roaming the streets. So, they want to apply the technology to let the visitor know more about the place.

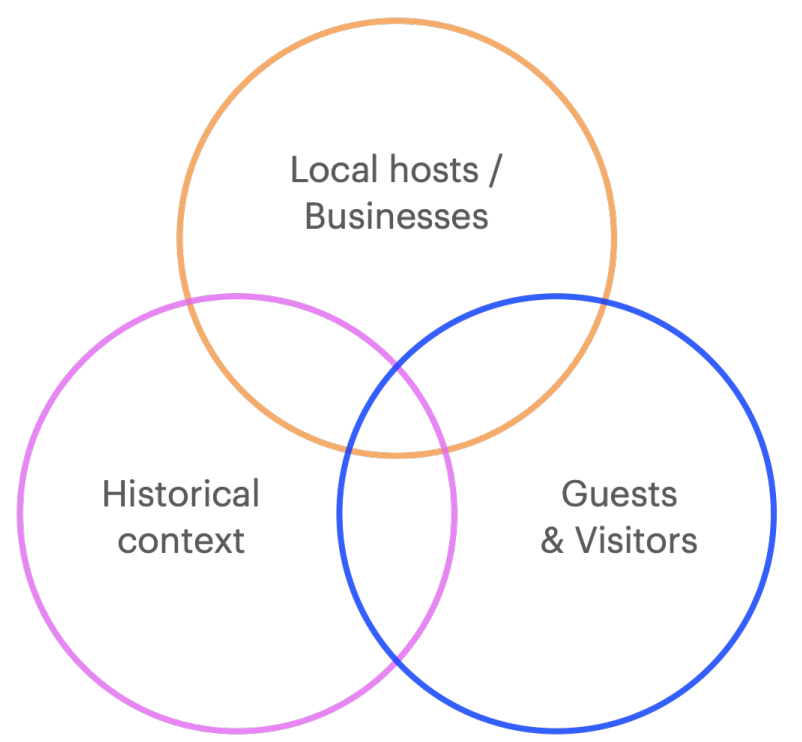

Figure. 3: Goal of the concept. (source from designer, drawn by this research)

The goal of this concept is to create a balance between all three pillars, to build a strong relationship between local businesses and historical context to encourage participation from guests. 
As you can see the scenario flow in Figure. 4, upon visiting Dadaocheng start your journey with the QR code located on the historical newsstand. Enabling your mobile device to become a window into the past using the AR scanner, you can engage with the nien-tai poststand which connect you with historical figures from 1920s past. Next, add as the friend of your LINE app and learn through the stories of the 1920s historical figures and discover more about rich culture and history by engaging in conversation with them. After returning home, continue to engage with Dadaocheng's history through 360 VR experiences to bring additional learning into the convenience of your own home, you can even share these experiences with your friends to continue engaging others.

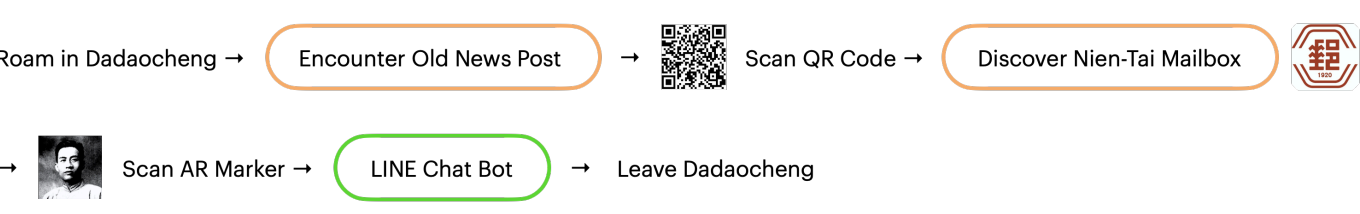

2

Return Home $\rightarrow$ Virtually visit Dadaocheng VR Museums $\rightarrow$ Share \& Promote Experiences

Figure. 4: Flow of the journey from the beginning of your arrived till you back home. (source from designer, drawn by this research)

Service touchpoint shows the relationship between the time of the journey and technologies (as Fig.5), they import different technologies at different stages to solve the corresponding problems, there're mainly three kinds of action, "research"- before you visit there, "discovery" when you roman on the street, and "interaction" - during the visiting or when you walk into the historical place, even back home to review the background story of the place you've visited.

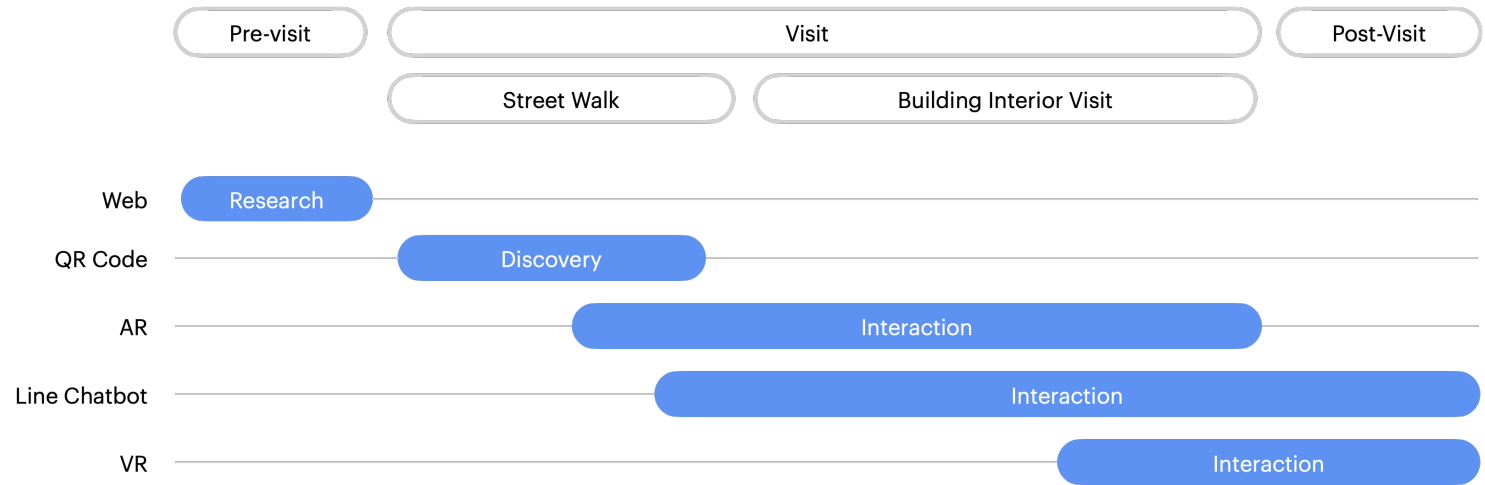

Figure. 5: Service touchpoint. (source from designer, drawn by this research)

\subsubsection{Move Your Bumbum (Berlin)}

Move your bum bum is a conceptual application, through data-collecting and visualize them through screens to encourage people to go out and have a deeper look in their neighborhood. This conceptual application will collect the data through the internet or your devices to suggest the locations which fit you well. Most of the system now recommend the popular place for user, but 
is that the place they really want to visit? Based on this question, they provide the new way for user to have a chance for visiting the new place.

Improving people's feelings of indifference to community by studying their thought, so they made the interview and draw the cognitive mapping to find out the reason that cause less interest to their community, as figure 6. Cognitive map is a type of mental representation which serves an individual to acquire, code, store, recall, and decode information about the relative locations and attributes of phenomena in their everyday or metaphorical spatial environment. The concept was introduced by Edward Tolman in 1948 [4], is any visual representation of a person's or a group's mental model for a given process or concept, it also diverse in nature and purpose, no restriction on structure or form [6].

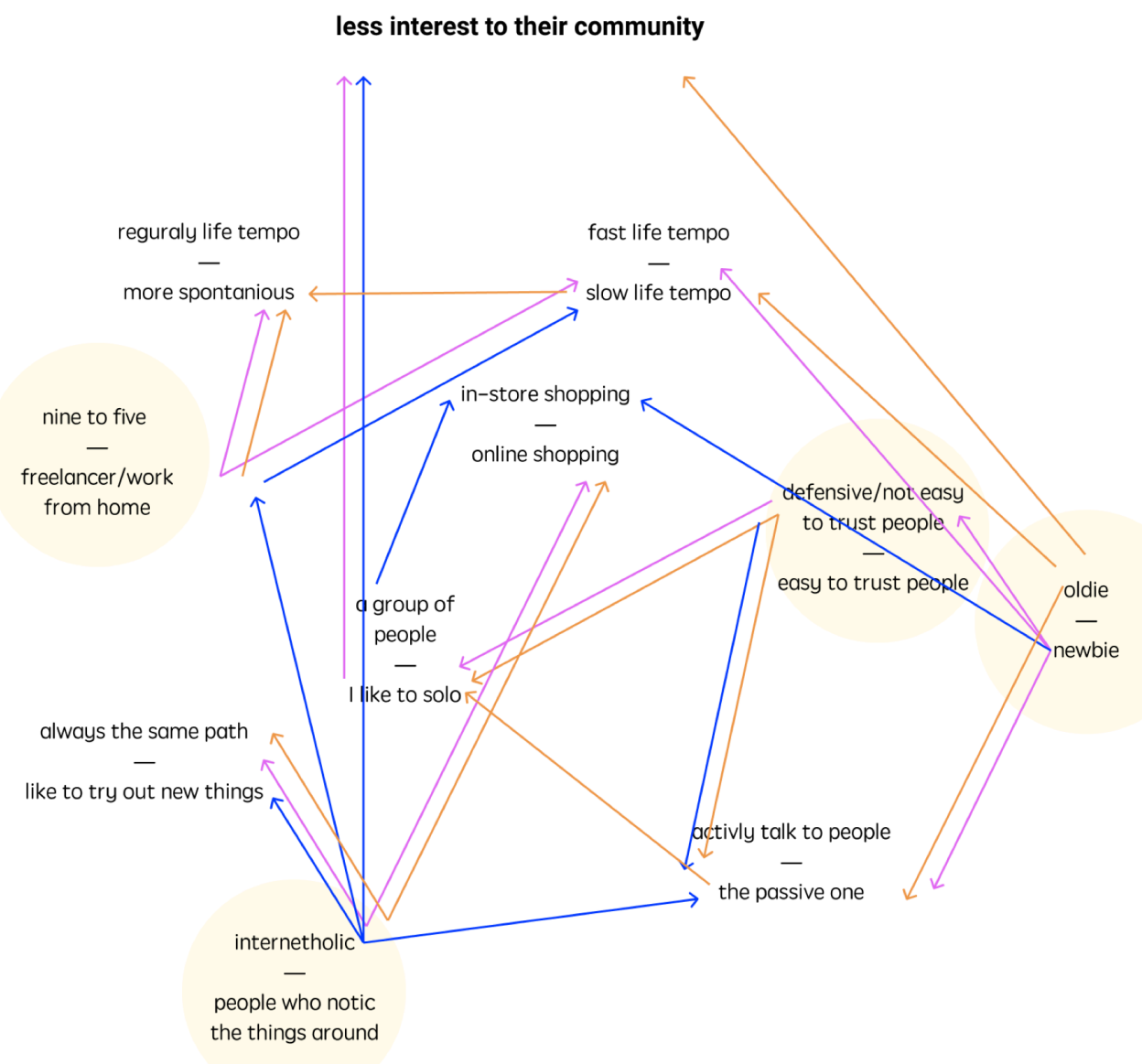

Figure. 6: cognitive mapping to make sure the problem (source from designers.)

Lack of emotion, lack of motivation to explore, and general lack of awareness of their living environment.

To increase motivation by increasing connections and generating interest through influencing human behavior. It comes out these four main functions, search, pin feedback and recommendation (Fig.7). "Search" is the basic function, during the learning process, the system needs more personal data to get to know the user more. You can see the first screen that shows the result and the filters, there's no ranking in this application, because they want people to explore different places not the popular places. Function "Pin" exists on the screens when it shows the 
map, you can pin it with camera and voice. while you are walking through the street, sometimes we will find out some interesting places where we would like to visit again or for next time, we will pin it. The "Feedback" here is only for your own, after visiting the places you have been to, a personal feedback can help you memorize the feeling and experiences. It also will help the system knows your preferences. The last one "Recommendation", system will recommend the places based on learning your preferences and trigger you to explore more places with empathy. Another example, you've pinned a cake store in your wish list, and the system knows through the calendar that your friend's birthday is coming up, so the system gives you a notification to suggests you, to take this opportunity for visiting.

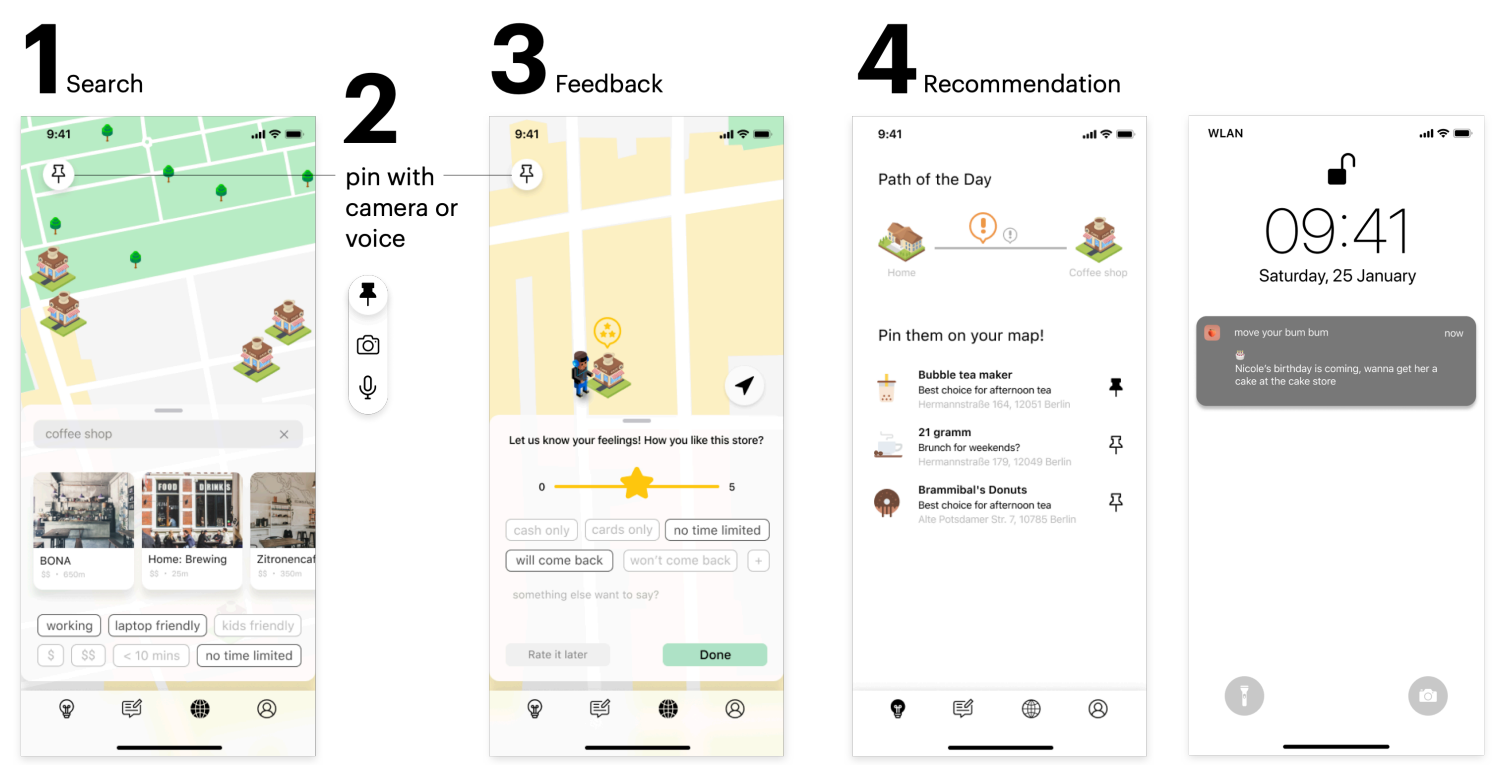

Figure. 7: Main screens of Move your bumbum. (source from designers.)

\subsection{Data Analysis}

\subsubsection{Analysis with Innovation Model}

We present the analysis results based on the innovation model with radar plots (as Fig.8).. In feasibility perspective "Bridging Conversations with the Past \& Present" want to build the authentic experience, so they used AR and VR to accomplish, also, web VR lower the threshold for development it performs quite well here. Move your bumbum's recommendation system must learn and provide advice in the desired way otherwise it would be a normal map application, so the performance here is lower than another. Desirability, culture background story had been considered in Bridging Conversations with the Past \& Present's context, although the interface is a bit simple, but the user has many interactions with it, it is fine of this perspective. Move your bumbum created by interface design background, focus more on the user behavior and user experience so the performance is higher then. The viability perspective, both cases put more attention on the user and technology, it might help with the local store but obviously, it's lacking business model and future framework. 


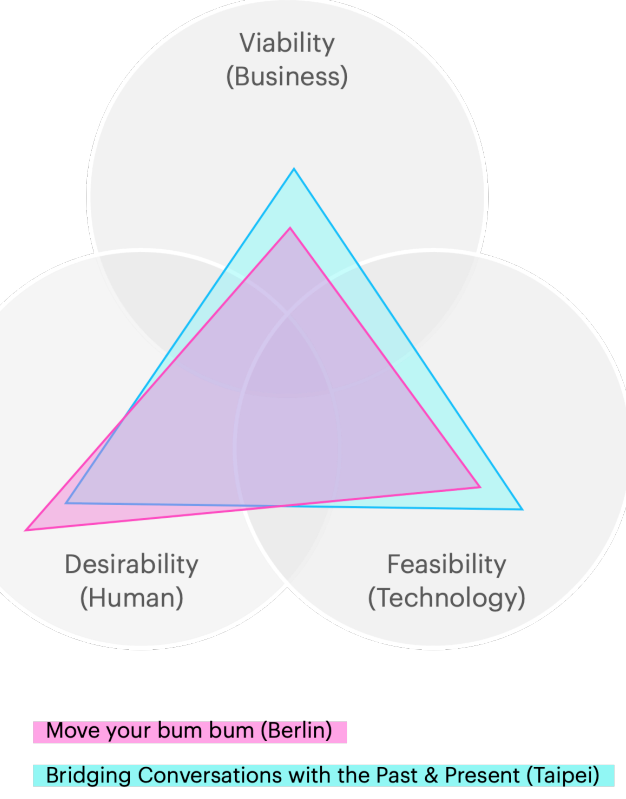

Figure. 8: Level of performance in three perspective. (source from designers.)

\section{Conclution}

Instead of traditional interpretation, there is no time limit for visiting of the concept "Bridging Conversations with the Past \& Present", and visitors can watch it anytime. Focus on the implementation of technology, using these tools to understand the historical and cultural background of a specific region, so that visitors can have a more in-depth experience and sense of presence. conceptually there is no specific user group restriction, but the current usage is not popular, so the application of web VR can lower the threshold of use. Move your bum bum is an exploratory app from the user's perspective, which envision the preferences of users in the process of use, to achieve the purpose of increasing stickiness, through the visiualisation to encourage user. At the same time, the recommendation system will also integrate the information more into your life, to let you become more aware of your surroundings by taking the active role in traveling to unknown places. It has no specific area restrictions, can be used anywhere. As the result of analysis by the innovation model, we need to not only balance integration of technology and design content but also include a business field to make the service system more complete, considering the size of the market can be more credible when actually implementing the development of product.

\section{Reflection \& Future Work}

For the future work, action research is not really strict, so to interview experts in different fields can be an efficient way to improve the theory. This research is a pilot study of different disciplines, which still needs to find more cases of similar topics and make the comparison more dependable, and also implement interdisciplinary to verify and improve the practice of this field of study. 


\section{References}

[1] David E. Avison, Francis Lau, Michael D. Myers, Peter Axel Nielsen, Action Research, Communications of the ACM Volume 42, Number 1 (1999), Pages 94-97

[2] Grady M.P., Qualitative and action research: A practitioner handbook (1998)

[3] J. Spencer Clark, Suzanne Porath, Julie Thiele, Morgan Jobe, Action research (2020)

[4] Tolman, Edward C. Cognitive maps in rats and men. Psychological Review. (1948), 55 (4): 189-208

[5] Melinda Kolk, Embrace Action Research - improve classroom practice with action research ... and tell the story, Creative Educator, https://dl.acm.org/doi/fullHtml/10.1145/291469.291479?casa token=fJ7udr0ZtowAAAAA:ljzNGEfo wtSAkWD8_FnbTUkN9tLhgDm7jXczzeYdI6gPX06UwkY-lvkL77k2wo6scATqPQjN8EMm4Cs

[6] Nielsen Norman Group, Cognitive Maps, Mind Maps, and Concept Maps: Definitions (2019)

[7] City Yeast http://www.cityyeast.com/about.php (2013) 